\title{
The galactic diffuse high energy neutrino flux
}

\author{
F.L. Villante* \\ L'Aquila University, Physics and Chemistry Department, 67100 L'Aquila, Italy \\ INFN, Laboratori Nazionali del Gran Sasso, 67100 Assergi (AQ), Italy \\ E-mail: villante@lngs.infn.it \\ C. Evoli \\ Gran Sasso Science Institute, 67100 L'Aquila (AQ), Italy \\ E-mail: carmelo.evoliegssi.infn.it

\section{G. Pagliaroli} \\ Gran Sasso Science Institute, 67100 L'Aquila (AQ), Italy \\ E-mail: giulia.pagliaroli@gssi.infn.it
}

\begin{abstract}
We calculate the diffuse high energy neutrino flux produced by the interactions of cosmic rays with the gas contained in our Galaxy. In order to quantify the role of uncertainties in the cosmic ray propagation models, we consider three different assumptions for the cosmic ray distribution in the Galaxy. We provide expectations for the spectrum and the angular dependence of the diffuse galactic neutrino flux. We compare our predictions with the isotropic flux required to explain the 54 HESE events observed by IceCube in four years data taking.
\end{abstract}

Neutrino Oscillation Workshop

4 - 11 September, 2016

Otranto (Lecce, Italy)

\footnotetext{
* Speaker.
} 


\section{Introduction}

In four years of data taking, the IceCube detector has detected 54 High Energy Starting Events (HESE) with deposited energy between $20 \mathrm{TeV}$ and $2 \mathrm{PeV}$ which are compatible with an astrophysical population of high energy neutrinos [1]. The observed excess has been also confirmed by independent observation of upward going passing muons in IceCube [2]. The origin of these neutrinos is still unknown and potential sources include supernova remnants, pulsars, active galactic nuclei and starburst galaxies. Dedicated searches for point-like or extended sources have been performed by IceCube [3]; however, at present, no significant clustering or correlation of event arrival directions with potential source distributions has been found, thus leaving open the possibility of a diffuse astrophysical neutrino population. In this short note, we review the recent calculation [4] of the high energy diffuse galactic neutrino flux.

\section{The diffuse galactic neutrino flux}

It is known that the interactions of Cosmic Rays (CR) with the interstellar medium is a guaranteed source of diffuse neutrinos in our Galaxy. The calculation of the neutrino flux at $E_{V} \simeq 100 \mathrm{TeV}$ is, however, quite uncertain because it requires the knowledge of the CR distribution at $E_{\mathrm{CR}} \simeq 20 E_{v}=2 \mathrm{PeV}$ in all the regions of the Galaxy where the gas density is not negligible. We consider here three different prescriptions of increasing complexity for relating the CR distribution $\varphi_{\mathrm{CR}}(r, E)$ to the local determination $\varphi_{\mathrm{CR}, \odot}(E)$. These are described below and shown in Fig.1:

Case A: We assume that CR density is homogenous in the Galaxy, so that the distribution of neutrino arrival directions is only determined by the gas column density;

Case B: We assume that the CR flux scales proportionally to the CR source density described by slight modification of the SNRs distribution of Green et al. [5], see [4] for details;

Case C: We consider that not only the spatial distribution of CR follows the CR source distribution but also the spectral index of CR depends on the galactocentric distance. This last case is intended to reproduce the trend of the spectral index with $r$ observed by [6] at $\bar{E} \simeq 20 \mathrm{GeV}$. Note that, being forced to rely on extrapolations from low energy data, even a relatively small change in the spectral index at $\bar{E}$ introduces a large variation of the high energy CR flux, as it is displayed in Fig.1.

For the three considered cases the flux of high energy neutrinos and antineutrinos of each flavour at Earth can be written as:

$$
\varphi_{v}\left(E_{v}, \hat{n}_{v}\right)=\mathscr{F}\left(E_{v}\right) \mathscr{I}\left(\hat{n}_{v}\right)
$$

where $\mathscr{I}=\mathscr{A}, \mathscr{B}, \mathscr{C}$ depends on the considered scenario. The neutrino energy distribution is described by the function:

$$
\mathscr{F}_{v}\left(E_{v}\right)=\frac{4 \pi}{3} \mathscr{N}_{\mathrm{H}} \int_{E_{v}}^{\infty} \frac{d E}{E} \sigma(E) F\left(\frac{E_{v}}{E}, E\right) \varphi_{\mathrm{CR}, \odot}(E),
$$

where $\mathscr{N}_{\mathrm{H}}=2.19 \times 10^{21} \mathrm{~cm}^{-2}$ is the average column density of the gas, $\sigma(E)$ is the total inelastic cross section, the adimensional distribution function $F\left(E_{v} / E, E\right)$ is given in [7] and we assumed that, due to neutrino mixing, the neutrino flux at Earth is equally distributed among the different 


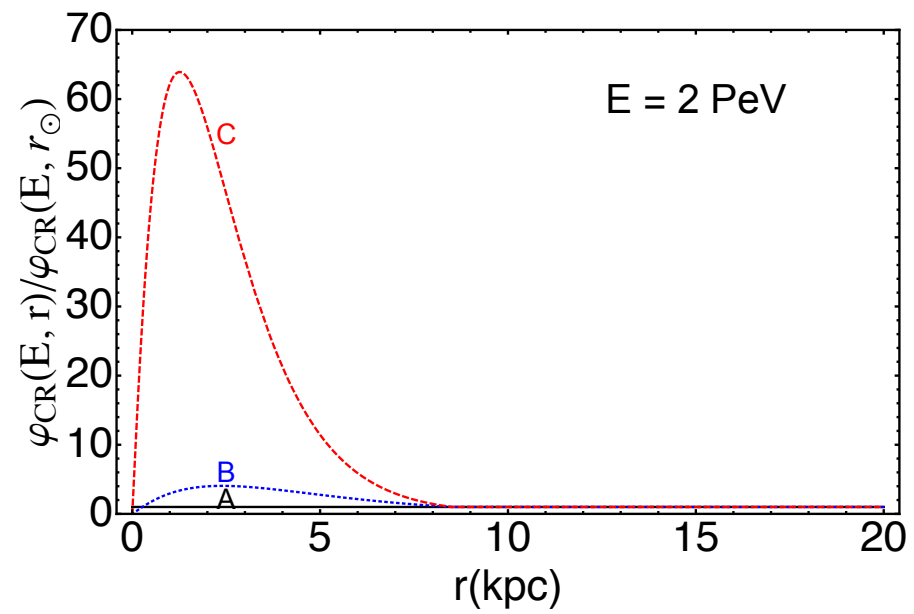

Figure 1: The CR flux at $E=2 \mathrm{PeV}$ as a function of the distance from the galactic center in the three considered scenarios. We assume that the CR flux is approximately constant along the galactic latitudinal axis. See text for details.

flavours. For neutrino energies $E_{v}=10 \mathrm{TeV}-1 \mathrm{PeV}$, this function is well approximated by:

$$
\mathscr{F}\left(E_{v}\right)=f\left[\frac{E_{v}}{100 \mathrm{TeV}}\right]^{-\alpha\left(E_{v}\right)}
$$

where $f=4.76 \times 10^{-7} \mathrm{GeV}^{-1} \mathrm{~m}^{-2} \mathrm{y}^{-1}$ and the spectral index is given by:

$$
\alpha\left(E_{V}\right)=2.65+0.13 \log _{10}\left(E_{V} / 100 \mathrm{TeV}\right) .
$$

The neutrino angular distribution is contained in the function $\mathscr{I}\left(\hat{n}_{v}\right)$ which is calculated in [4] for the three considered scenarios. The angle-integrated neutrino fluxes are given by:

$$
\phi_{v}\left(E_{v}\right)=\overline{\mathscr{I}}_{\mathscr{F}_{v}}\left(E_{v}\right)
$$

with the factors $\overline{\mathscr{I}} \equiv \int d \Omega \mathscr{I}(\hat{n})$ equal to $\overline{\mathscr{A}}=1, \overline{\mathscr{B}}=1.23$ and $\overline{\mathscr{C}}=2.34$, respectively. The integrated fluxes can be compared with the isotropic flux:

$$
\mathscr{F}_{\text {iso }}\left(E_{V}\right)=f_{\text {iso }}\left[\frac{E_{V}}{100 \mathrm{TeV}}\right]^{-2.58}
$$

where $f_{\text {iso }}=8.72 \times 10^{-6} \mathrm{GeV}^{-1} \mathrm{~m}^{-2} \mathrm{y}^{-1}$, that corresponds to the HESE event rate observed by IceCube in four years data taking [1]. At the neutrino energy $E_{v}=100 \mathrm{TeV}$ that provides the most relevant contribution to the HESE data sample, the diffuse galactic neutrino component is equal to $5 \%, 7 \%$ and $13 \%$ in Case $A, B$ and $C$ respectively, of the isotropic flux required to explain the 54 events observed by IceCube. This component is thus not negligible but always subdominant.

The angular dependence of the flux in the three considered scenarios is shown in Fig. 2 as a function of the galactic longitude $l$ (upper panel) and latitude $b$ (lower panel). We note that: i) In the considered scenarios, it always exists a region, that contains the galactic center, where the 

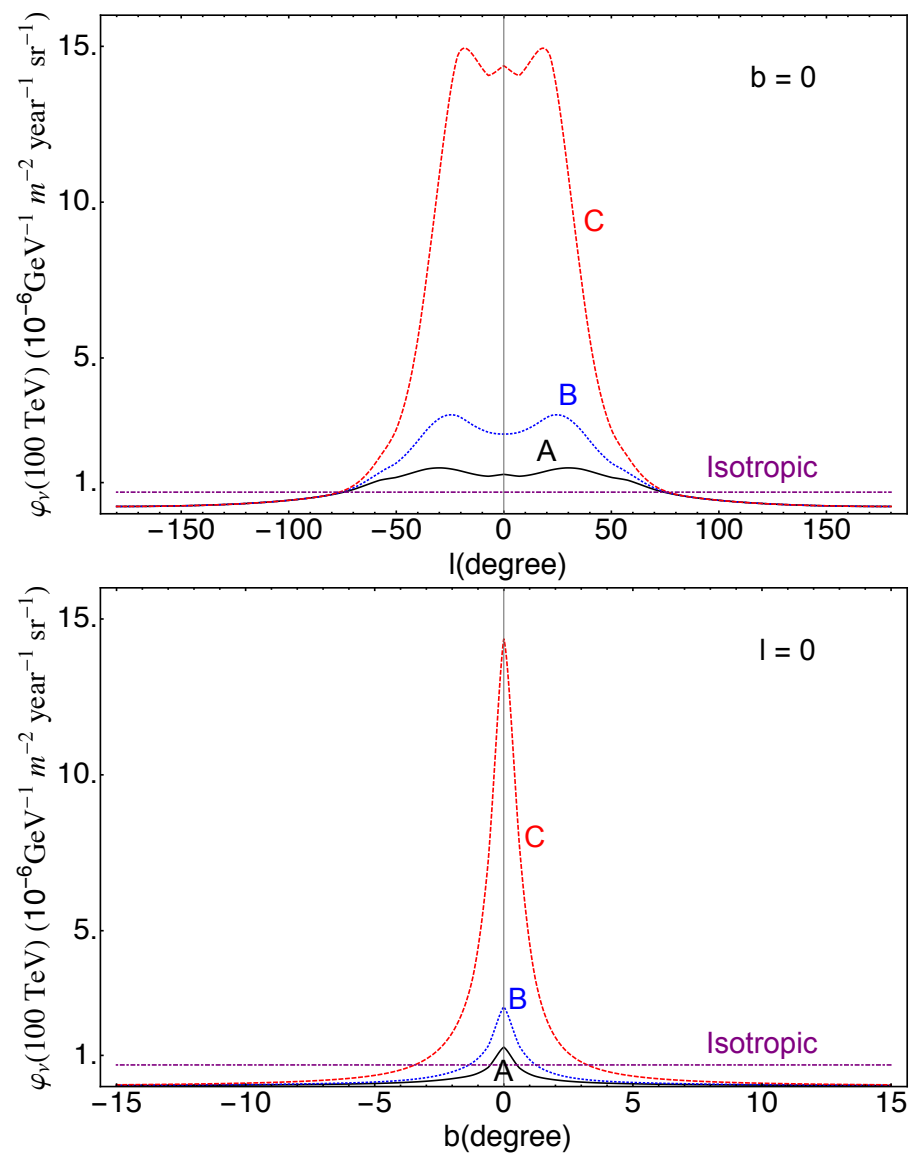

Figure 2: The neutrino flux at $E_{v}=100 \mathrm{TeV}$ as a function of the Galactic longitude (upper panel) and latitude (lower panel) for the three different models considered for CR distribution. The solid black line corresponds to Case A, the blue dotted line corresponds to Case $B$ and the red dashed line corresponds to Case $C$. The isotropic flux that reproduces IceCube HESE data is also reported for comparison with a purple dot-dashed line.

neutrino flux produced by CR interacting with the gas contained in the galactic disk, is comparable or larger than the isotropic contribution. Thus, the diffuse galactic neutrino component is, in principle, sufficiently intense to be detected;

ii) The region where the diffuse galactic neutrino component dominates is quite narrow. Even in the most optimistic Case $C$, the region where $\varphi_{v}\left(E_{v}, \hat{n}_{v}\right) \geq \mathscr{F}_{\text {iso }}\left(E_{v}\right) / 4 \pi$ corresponds to $|l| \leq 70^{\circ}$ and $|b| \leq 3^{\circ}$. Thus, the optimal detector should have a good pointing capability in order to avoid diluting the signal below the isotropic background. Unfortunately, the IceCube HESE data set is dominated by showers events that do not allow to reconstruct the neutrino arrival direction with sufficient accuracy. It was shown in [4] that only Case C has a non negligible chance of detection, due to the fact that it predicts a larger and more pronounced emission from regions close to the galactic center. In the optimal region of the sky given by $|l| \leq 60^{\circ}$ and $|b| \leq 15^{\circ}, \sim 2.5 \mathrm{HESE}$ events are expected in four years of data-taking that could be observed with $\sim 1 \sigma$ significance above the isotropic contribution. 
iii) The angular distributions are quite different in the three considered cases. The maximal emission is always achieved for $l \simeq \pm 25^{\circ}$ and $b=0^{\circ}$ but the neutrino fluxes may differ by large factors for $|l| \leq 90^{\circ}$. To be quantitative, the flux from the galactic center is larger by a factor $\sim 2$ and $\sim 11$ in Case $B$ and $C$ respectively, with respect to the value obtained in the assumption of uniform CR density (i.e. Case A). In perspective, this could provide an handle to discriminate among different scenarios, in an ideal detector with sufficient statistics and good pointing capability.

\section{References}

[1] M. G. Aartsen et al. [IceCube Collaboration], arXiv:1510.05223 [astro-ph.HE]

[2] M. G. Aartsen et al. [IceCube Collaboration], Phys. Rev. Lett. 115 (2015) no.8, 081102

[3] M. G. Aartsen et al. [IceCube Collaboration], arXiv:1605.00163 [astro-ph.HE]

[4] G. Pagliaroli, C. Evoli and F. L. Villante, JCAP 1611 (2016) no.11, 004

[5] D. A. Green, Mon. Not. Roy. Astron. Soc. 454 (2015) no.2, 1517

[6] F. Acero et al. [Fermi-LAT Collaboration], Astrophys. J. Suppl. 223 (2016) no.2, 26

[7] S. R. Kelner and F. A. Aharonian, Phys. Rev. D 78 (2008) 034013 Erratum: [Phys. Rev. D 82 (2010) 099901] 